\title{
Erythropoietin-Receptor Gene Regulation in Neuronal Cells
}

\author{
IWONA WALLACH, JUAN ZHANG, ANIKA HARTMANN, FRANK K.H. vAN LANDEGHEM, ANNA IVANOVA, MARTIN KLAR, \\ AND CHRISTOF DAME
}

\author{
Department of Neonatology [I.W., J.Z., A.H., A.I., M.K., C.D.], and Institute of Neuropathology [F.K.H.L.], Charité Universitätsmedizin \\ Berlin, Berlin D-13353, Germany
}

\begin{abstract}
Because erythropoietin (Epo) is intensively studied as neuroprotective agent, Epo receptor (EpoR) regulation in neurons is of particular interest. Herein, we investigated molecular mechanisms of EpoR regulation in neuronal cells including the role of GATA transcription factors. First, developmental downregulation of EpoR expression in murine brain was observed. A differential expression pattern of the Gata factors was found in these specimens as well as in murine adult neural stem cells (NSC) and primary rat neurons, astrocytes, and microglia. Human SH-SY5Y cells served as a model to analyze EpoR regulation. In vitro binding of GATA-2, -3, and -4 to the 5 '-flanking region was demonstrated. In reporter gene assays, the activity of a region containing two GATA binding sites was significantly induced when these GATA factors were overexpressed. However, GATA factors alone did not affect endogenous EpoR expression. Importantly, EpoR transcripts have doubled under hypoxia. Furthermore, we analyzed the methylation pattern close to the GATA motifs. Indeed, demethylation with 5-Aza-2'-deoxycytidine (Aza) resulted in upregulation of EpoR mRNA. Additionally, several CpGs were mostly nonmethylated in SH-SY5Y cells, but methylated in specific regions of the human adult brain. Thus, methylation may be involved in developmental EpoR downregulation. (Pediatr Res 65: 619-624, 2009)
\end{abstract}

$\mathrm{R}^{\mathrm{e}}$ ecombinant erythropoietin (rEpo) exhibits significant neuroprotective effects in animal studies of neonatal and adult brain injury and in the first clinical trials (1). REpo mediates its neuroprotective effects by binding to the erythropoietin receptor (EpoR) and by activating complex signaling cascades (2). The requirement of EpoR signaling for brain development has been demonstrated in EpoR null mice and in mice with selective EpoR ablation in the brain, which have a reduced number of neuronal progenitor cells in the subventricular zone and show extensive apoptosis in the brain (3-5). Although the absolute level of EpoR expression is developmentally downregulated, expression persists in selected areas, and vascular endothelial cells as well as mature neurons $(4,6)$. In fact, EpoR expression is also downregulated with differentiation of neural progenitor cells (5). Impaired poststroke neurogenesis in adult mice with brain-specific EpoR deletion

Received September 29, 2008; accepted January 8, 2009.

Correspondence: Christof Dame, M.D., Department of Neonatology, Campus Virchow-Klinikum, Charité-Universitätsmedizin Berlin, Augustenburger Platz 1, D-13353 Berlin, Germany; e-mail: christof.dame@ charite.de

Supported by the Jürgen Manchot Stiftung, doctoral fellowship from the HumboldtUniversität Berlin/Charité-Universitätsmedizin Berlin (I.W.), the Deutsche Forschungsgemeinschaft (DA 484/2-1) (C.D.), and a grant from the Roche Foundation for Anaemia Research (ID 5463534641).

Current address (FKHL): Institute of Neuropathology, Universitätsklinikum Bonn, Sigmund-Freud-Strasse 25, D-53105 Bonn, Germany. confirmed the remaining functional implication of EpoR expression (4). For using rEpo as neuroprotective agent, understanding of the regulation of EpoR expression therefore becomes extremely important, even if erythropoietin (Epo) can mediate neuroprotection partially through a heterodimer composed of one EpoR monomer and one common $\beta$-subunit homodimer (7). Beside the fact that EpoR expression in neuronal cells is upregulated in response to hypoxia and TNF- $\alpha(3,8-10)$, the molecular mechanisms of EpoR regulation in the nervous system are almost unknown.

Analysis of the EpoR 5'-flanking region revealed several potential GATA binding sites. The transcription factor GATA-1 activates EpoR expression in hematopoietic cells, but is not expressed in neuronal cells (11). Thus, the question arises whether other GATA transcription factors are involved in EpoR regulation in the nervous system. GATA transcription factors are a family of six zinc-finger proteins, which bind to the (T/A)GATA(G/A) consensus sequence and play important roles in cellular differentiation and proliferation. Despite similarities in the binding sites and structure, different GATA factors take over specific functions, likely depending on surrounding sequences and on cofactors. A first attempt to link GATA transcription factors to EpoR expression in neuronal cells revealed that stable overexpression of GATA-3 (using a vector construct that also expresses $\mathrm{Sp} 1$ ) resulted in increased EpoR expression in human teratocarcinoma-derived neuronal precursor cells (NT2) (3). REpo stimulated GATA-3 expression in NT2 cells, suggesting that neuronal GATA transcription factors could be involved in a regulatory network allowing rEpo to enhance EpoR expression (3).

The aims of the study were (1) to elucidate the functional relevance of the GATA motifs for EpoR regulation in cells with characteristics of neuronal precursors and (2) to gain information on mechanisms involved in the developmental downregulation of the EpoR in the brain.

\section{MATERIALS AND METHODS}

Preparation of murine tissues and cells. WT CD61 mice were mated and embryos were collected at embryonic day (E) E9.5/E11.5/E13.5 and neonatal day 1. For RNA preparation, tissue specimens were homogenized in TRIZOL (Invitrogen, Karlsruhe, Germany).

Adult neural stem cells (NSC) were prepared from B16C57 mice as described (12). NSC were cultivated in NBMA (Invitrogen) containing 2\%

\footnotetext{
Abbreviations: Aza, 5-Aza-2'-deoxycytidine; E, embryonic day; Epo, erythropoietin; EpoR, erythropoietin receptor; FOG, friend-of-GATA; NSC, neural stem cells; nt, nucleotide(s); rEpo, recombinant Epo
} 
Table 1. Sequences of RT-PCR primers

\begin{tabular}{|c|c|c|c|}
\hline Gene & Forward primer $(\mathrm{FW})$ & Reverse primer (RV) & Product size (bp) \\
\hline$h \beta$-actin & gctcgtcgtcgacaacggctc & caaacatgatctgggtcatcttctc & 353 \\
\hline$m \beta$-actin & actgctctggctcctagcac & acatctgctggaaggtggac & 115 \\
\hline$r \beta$-actin & atcgtgggecgecctagcacc & ctctttaatgtcacgcacgatttc & 542 \\
\hline hEpoR & accgtgtcatccacatcaat & gccttcaaactcgctctctg & 485 \\
\hline mEpoR & ggacacctacttggtattgg & gacgttgtaggctggagtcc & 451 \\
\hline rEpoR & ctatggctgttgcaacgcga & ccgagggcacaggagcttag & 401 \\
\hline$h G A P D H$ & aaggtcatccatgacaactt & ttcagctcagggatgacctt & 191 \\
\hline hGATA-1 & gatcctgctctggtgtcctcc & acagttgagcaatgggtacacc & 192 \\
\hline$h G A T A-2$ & ccctaagcagcgcagcaagac & gatgagtggtcggttctggcc & 164 \\
\hline mGata-2 & acacaccaccegatacccacctat & cctagcccatggcagtcaccatgc & 720 \\
\hline rGata-2 & acacaccacccaatacccacc & gcccatggcagtcaccatgc & 717 \\
\hline$h G A T A-3$ & gtacagctccggactcttccc & ctgctctcctggctgcagaca & 257 \\
\hline mGata-3 & acgtctcactctcgaggcagcatg & gaagtcctccagcgegtcatgcac & 566 \\
\hline rGata-3 & ccgtcctactacggaaactc & agttcacacactccetgect & 619 \\
\hline$h G A T A-4$ & ctccttcaggcagtgagagcc & ggtccgtgcaggaatttgagg & 368 \\
\hline mGata-4 & aggcgagatgggacgggacactac & cggcgggaagcggacag & 113 \\
\hline rGata-4 & gatgggacaggacactacct & acctgctggtgtcttagattt & 308 \\
\hline$h G A T A-5$ & tcgccagcactgacagctcag & tggtctgttccaggctgttcc & 290 \\
\hline$h G A T A-6$ & ttctaactcagatgattgcag & gctgcacaaaagcagacacga & 300 \\
\hline
\end{tabular}

All primers are given in the $5^{\prime}$ to $3^{\prime}$ direction.

$\mathrm{h}$, human; m, mouse; r, rat.

B27 w/o retinoic acid (Invitrogen), 1\% L-glutamine, $10 \mathrm{ng} / \mathrm{mL}$ bFGF, and 20 ng/mL EGF (Biochrom, Berlin, Germany).

Preparation of rat primary cells. Primary neuronal cultures of cerebral cortex from Wistar rat embryos (E17) were prepared as described (13). Astroglial and microglial cell cultures were prepared from newborn rats as described (14). Microglial cells were seeded at a density of 100,000 cells $/ \mathrm{cm}^{2}$ and harvested after $24 \mathrm{~h}$. Astrocytes were seeded at density of 120,000 cells $/ \mathrm{cm}^{2}$ and harvested after $48 \mathrm{~h}$. All animal studies were approved by the Institutional Review Board (Landesamt für Gesundheit und Soziales, Berlin).

Human tissues. Human adult tissue specimens obtained from the enthorinal, frontal and motor cortex, and cervical spinal cord were taken at post mortem examination with approval of the Institutional Review Board of the Charité after obtaining informed consent in accordance with the Berlin autopsy law.

Cell culture. The human neuroblastoma-derived cell line SH-SY5Y (ATCC; CRL-2266) was cultivated in RPMI 1640 with L-glutamine (Invitrogen), supplemented with penicillin/streptomycin and 10\% FCS. To achieve hypoxia, cells were incubated at $2 \% \mathrm{O}_{2}$ for $72 \mathrm{~h}$. In additional experiments, cells were treated with $5 \mathrm{U} / \mathrm{mL}$ rEpo (Epoetin-beta, Hoffmann-La Roche, Grenzach-Wyhlen, Germany) for $72 \mathrm{~h}$.

RNA isolation, RT-PCR, and real-time PCR. RNA was isolated using TRIZOL. First-strand cDNA was synthesized from $2 \mu \mathrm{g}$ of RNA using Oligo(dT) ${ }_{15}$ primer (Promega, Mannheim, Germany). Genes of interest were amplified using specific primers (Table 1 ). $\beta$-actin primers were purchased (Invitrogen, Cat. No. 10929-016). PCRs were repeated at least three times. PCR-products were resolved on $1.5 \%$ agarose gels. The photographs were inverted. For EpoR and $\beta$-actin quantification, TaqMan real-time PCR was performed using commercial primers (hEpoR Hs00181092_m1; h $\beta$-actin Hs99999903_m1; Applied Biosystems, Foster City, CA).

Protein preparation and Western blot analysis. Nuclear protein extracts were prepared and quantified as described (15). For Western blot analysis, proteins were electrophoresed on Novex 4-20\% Tris-glycine gels (Invitrogen) under denaturing conditions. The following primary antibodies (Santa Cruz Biotechnology, Santa Cruz, CA) were used: GATA-2 (CG2-96), GATA-3 (HG3-31), and GATA-4 (C-20).

Electrophoretic mobility shift assays. Electrophoretic mobility shift assay was performed as described (16). End-labeled double-stranded oligonucleotides (Table 2) were incubated with $8 \mu \mathrm{g}$ of SH-SY5Y nuclear extracts. In competition experiments, 400-fold excess of cold WT or mutant competitors was added. Where indicated, GATA-2 (CG2-96), GATA-3 (HG3-31), GATA-4 (C-20), or GATA-6 (C-20) antibodies (Santa Cruz Biotechnology) were postincubated with the samples for $20 \mathrm{~min}$ at $30^{\circ} \mathrm{C}$. DNA-protein complexes were separated on $5 \%$ polyacrylamide gels and visualized by autoradiography.

Expression plasmids, and transient transfections. Full-length sequences of human GATA-2 and GATA-3 were subcloned from pRSV/neo+GATA-2 and RSV/hGATA3 (provided by C.T. Noguchi, National Institutes of Health, and J.D. Engel, University of Michigan, respectively) into the pcDNA3.1(+)
Table 2. List of the oligonucleotides that have been used for electrophoretic mobility shift assay (EMSA) experiments based on the presence of predicted GATA binding site in the 5'-flanking region of the human EpoR gene (GenBank accession no. S45332)

\begin{tabular}{ll}
\hline Oligonucelotide & \multicolumn{1}{c}{ Single strand sequence } \\
\hline$-1504 /-1475$ & $5^{\prime}$-GTGCAGTGGCCAAATATCGGCTCACTGAAA-3' \\
$-538 /-509$ & 5'-AGGTGCAGTGGGAAGATTGCTTCAGTCCAG-3' \\
$-501 /-472$ & $5^{\prime}$-AGTGAGCTATGATTGTGCCACTGCACTCCA-3' \\
$-286 /-257$ & $5^{\prime}$-GCTGATTTCTGCGATAAAATCAGTAGGTAC-3' \\
mut $-286 /-257$ & $5^{\prime}$-GCTGATTTCTGCAAAAAAATCAGTAGGTAC-3' \\
$-54 /-25$ & $5^{\prime}$-AGGCACTTATCTCTACCCAGGCTGAGTGCT-3' \\
mut -54/-25 & 5'-AGGCACTCCCCTCTACCCAGGCTGAGTGCT-3' \\
$+120 /+149$ & 5'-GTGGCGGGGGCTGTATCATGGACCACCTCG-3' \\
\hline
\end{tabular}

Only the noncoding strand is shown; the potential GATA binding site is underlined; numbers indicate positions relative to the transcription start site. mut, mutant.

vector (Invitrogen). Full-length sequence of human GATA-4 (GenBank AY740706) was cloned into pcDNA3.1(+) vector. Expression vectors for human -2 friend-of-GATA-2 (FOG-2; pcDNA1/hFOG-2) and Sp1 (pN3-Sp1FLcomplete + empty vector $\mathrm{pN} 3$ ) were provided by E.N. Olson (University of Texas) and G. Suske (University of Marburg, Germany), respectively.

For stable overexpression of GATA-2, -3, and -4, SH-SY5Y cells were transfected with the expression plasmid using FuGENE 6 transfection reagent (Roche Diagnostics, Mannheim, Germany). Transfected cells were grown in selection medium containing geneticin G-418 (Invitrogen) for several weeks.

For transient overexpression of transcription factors, SH-SY5Y cells were transfected with the expression plasmid and harvested after $48 \mathrm{~h}$ with TRIZOL.

RNAi. RNAi for GATA-2 and -3 was performed using Silencer PreDesigned siRNA (Ambion, Austin, TX; GATA-2 ID no. 145419; GATA-3 ID no. 43336; Silencer Negative Control no. 1 siRNA). RNAi for GATA-4 was performed using pSilencer/hGATA-4 (852-870) vector and empty plasmid pSilencer 1.0-U6 from Ambion (15). SH-SY5Y cells, cultured without antibiotics in 6-well-plates, were transfected with 100 pmol siRNA/well or with $4 \mu \mathrm{g}$ of vector DNA/well using Lipofectamine 2000 (Invitrogen). Ninety-six hours after transfection, cells were harvested with TRIZOL.

Reporter gene assays. Fragments at nucleotides (nt) $-449 /+33$ and $-75 /+33$ (numbers indicate the position in the human EpoR 5'-flanking region relative to transcription start site, GenBank S45332) were amplified from human genomic DNA and cloned into pGL2-basic luciferase vector (Promega). Mutant construct GATA mut -44 (TATC to CCCC) was generated by Overlap-Extension-PCR.

For reporter gene assays, SH-SY5Y cells were transfected with $1 \mu \mathrm{g}$ pGL2-construct and $1 \mu \mathrm{g}$ expression vector for GATA-2, -3, -4 with or 
A

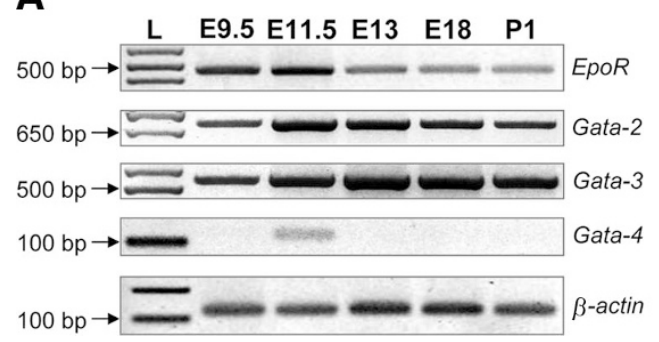

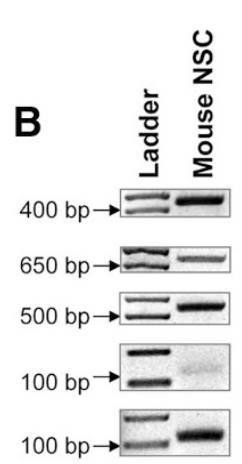

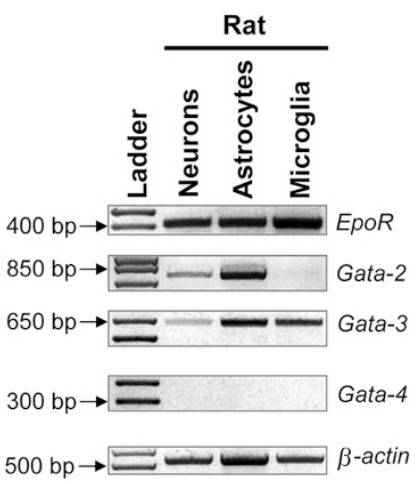

Figure 1. Expression of EpoR and Gata-2/-3/-4 in the CNS and brainderived primary cell types. (A) mRNA expression analysis during murine brain development. EpoR/GATA-2/-3/ $-4=28$ cycles; $\beta$-actin $=19$ cycles. $L$, ladder, $P 1$, neonatal day 1 . (B) mRNA expression in mouse adult NSC and in rat primary neurons, astrocytes and $\mathrm{mi}-$ croglia cells. EpoR/GATA-2/-3/-4 = 33 cycles; $\beta$-actin $=19$ cycles. without $1 \mu \mathrm{g}$ of pcDNA1/hFOG-2 or empty plasmid pcDNA3.1(+). After $48 \mathrm{~h}$, cells were harvested and analyzed for luciferase activity using the Luciferase Assay System protocol (Promega).

Methylation analysis. To investigate the effect of demethylation of genomic DNA on EpoR expression, SH-SY5Y cells were treated with 5 or 10 $\mu$ M 5-Aza-2'-deoxycytidine (Aza; Sigma Chemical Co., Saint Louis, MO). After 24 and $48 \mathrm{~h}$, the medium was changed and fresh Aza was added. $72 \mathrm{~h}$ after seeding, cells were harvested with TRIZOL. For methylation analysis, genomic DNA was prepared from SH-SY5Y cells $\left(6 \times 10^{6}\right)$ and human tissues (25 mg each) using the DNeasy Tissue Kit (Qiagen, Hilden, Germany). A total of $0.6-2 \mu \mathrm{g}$ of digested DNA were modified by bisulfite treatment using the EpiTect Bisulfite Kit (Qiagen). The EpoR region spanning nt -325 to +262 was amplified using HotStarTaq Master Mix Kit (Qiagen) with the primers IP50 $+\left(5^{\prime}\right.$-aaaaaataatttttatttatttcc- $\left.3^{\prime}\right)$ and IP55- (5'-actcatccttacctttactc- $\left.3^{\prime}\right)$. PCR was carried out with initial denaturation at $95^{\circ} \mathrm{C}$ for $15 \mathrm{~min}$, followed by 46 cycles of $95^{\circ} \mathrm{C}$ for $45 \mathrm{~s}, 50^{\circ} \mathrm{C}$ for $1 \mathrm{~min}, 72^{\circ} \mathrm{C}$ for $1 \mathrm{~min}$, and final extension at $72^{\circ} \mathrm{C}$ for $10 \mathrm{~min}$. PCR products were cloned into pCR2.1TOPO vector using TOPO TA Cloning Kit (Invitrogen). Six clones of each sample were verified by sequencing.

Statistical analysis. Before analysis, data were proven for normal distribution; statistical significance was examined by student $t$ test or one-way ANOVA post hoc comparisons as indicated. A $p$ value $<0.05$ was considered to be significant.

\section{RESULTS}

Expression of EpoR and Gata transcription factors in mammalian brain and its specific cell types. RT-PCR analysis of murine brain specimens confirmed developmental downregulation of EpoR (Fig. 1A). We detected EpoR mRNA in murine adult NSC and in rat primary neurons, astrocytes, and microglia (Fig. 1B). Moreover, we found expression of Gata-2 and -3 throughout gestation and in adult NSC. Although Gata-3 was expressed in rat neurons, astrocytes, and microglia, we detected Gata-2 only in rat neurons and astrocytes. A faint Gata-2 band in microglia resulted from contamination of this culture with astrocytes. Weak Gata-4 mRNA expression was detected only in brains from E11.5 mouse embryos and in adult murine NSC (Fig. 1).

SH-SY5Y cells as model to study nonhematopoietic EpoR regulation. We analyzed expression of all GATA transcription factors in SH-SY5Y cells because this cell line has previously been established as model for studying mechanisms of neuron-specific, hypoxia-inducible Epo gene expression, and expresses functional "classical" EpoR in the absence of the common $\beta$-subunit $(17,18)$. Figure $2 A$ shows mRNA expression of GATA-2, -3 , and -4 as well as of EpoR. In contrast, GATA-1, -5 , and -6 were not expressed. The expression of GATA-2, -3 , and -4 protein was confirmed in nuclear protein extracts (Fig. 2A).
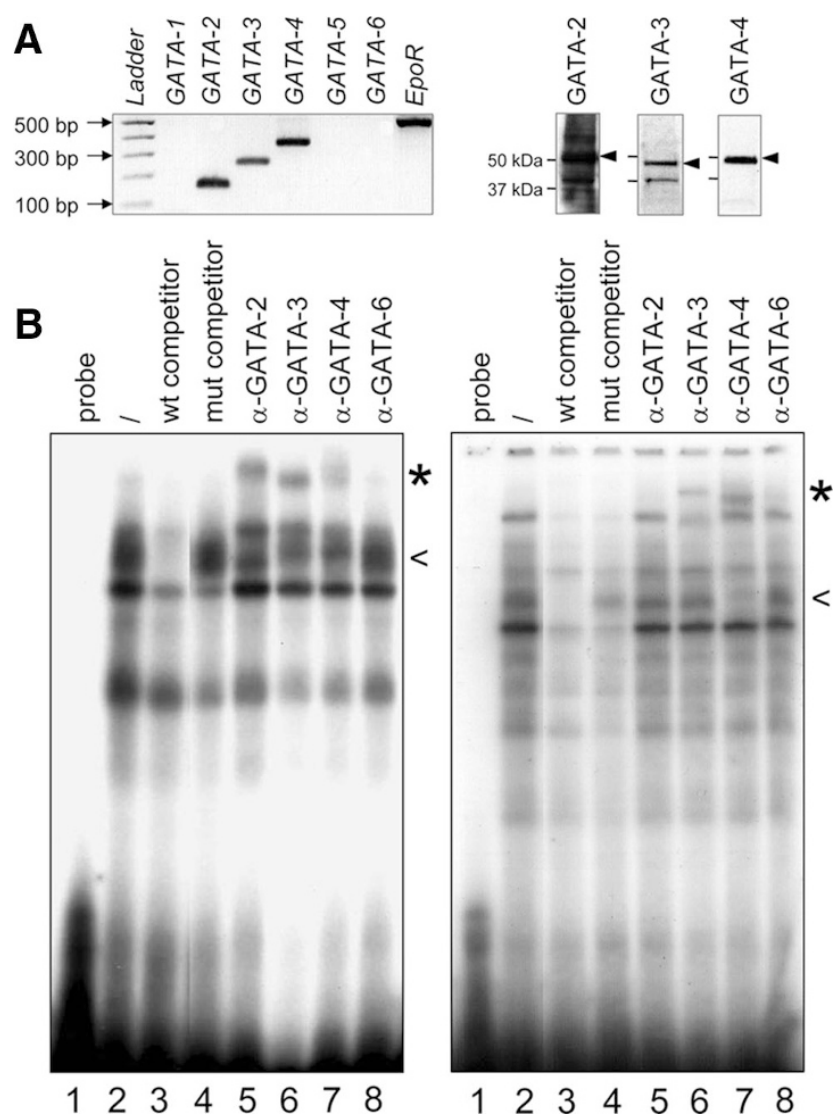

Figure 2. Interaction of GATA transcription factors with the human EpoR promoter region in SH-SY5Y cells. (A) Left panel: RT-PCR analysis (33 cycles) of EpoR and GATA transcription factor expression. Right panel: Western blot analysis of GATA-2/-3/-4 in nuclear protein extracts. (B) Electrophoretic mobility shift assays of the EpoR 5'-flanking region (left: $-286 /-257$; right: $-54 /-25)$. Protein-complexes specific for binding to the GATA site are indicated by an arrowhead, super-shifts with a star.

In vitro binding of GATA-2, -3 , and -4 to the EpoR 5 '-flanking region. Sequence analysis showed six potential binding sites in the EpoR 5'-flanking region, located at nt $-1490 /-1487,-524 /-520,-491 /-487,-274 /-271,-47 /$ -44 , and $+133 /+136$. Incubation of oligonucleotides containing the $-274 /-271$ and $-47 /-44$ GATA sites with nuclear proteins from SH-SY5Y cells led to formation of several protein-DNA complexes (Fig. 2B, lane 2). Although all complexes diminished significantly after preincubation with excess of WT competitor oligonucleotides (lane 3), one distinct 
protein-DNA complex remained after preincubation with a mutant oligonucleotide (lane 4), suggesting that formation of this protein-DNA complex requires the GATA site. Incubation with specific antibodies (lanes 5-7) resulted in super-shifted complexes, indicating binding of GATA-2, -3 , and -4 to the $-286 /-257$ oligonucleotide (Fig. 2B, left panel), and of GATA-3 and -4 to the $-54 /-25$ oligonucleotide (right panel). Incubation with a GATA-6 antibody (lane 8) served as negative control because GATA-6 is not expressed in SH-SY5Y cells. No specific interaction of GATA factors was found at the other four GATA motifs.

Effects of forced hGATA-2, -3, and -4 expression on EpoR promoter activity and EpoR expression. The effects of overexpression of hGATA-2, -3 , and -4 on the activity of two reporter gene constructs $(-449 /+33$ and $-75 /+33)$ are presented in Fig. $3 A$. Both constructs were activated to a similar extent by hGATA-2 and -3 (2.6- to 2.9-fold, $p<0.01$ ), and somewhat higher by hGATA-4 (3.5- to 4.8-fold, $p<0.01$ ). Unexpectedly, mutation of the $-47 /-44$ GATA binding site did not abolish reporter gene activation. To prove whether the cofactor FOG-2 is implicated in activation of EpoR by GATA

A
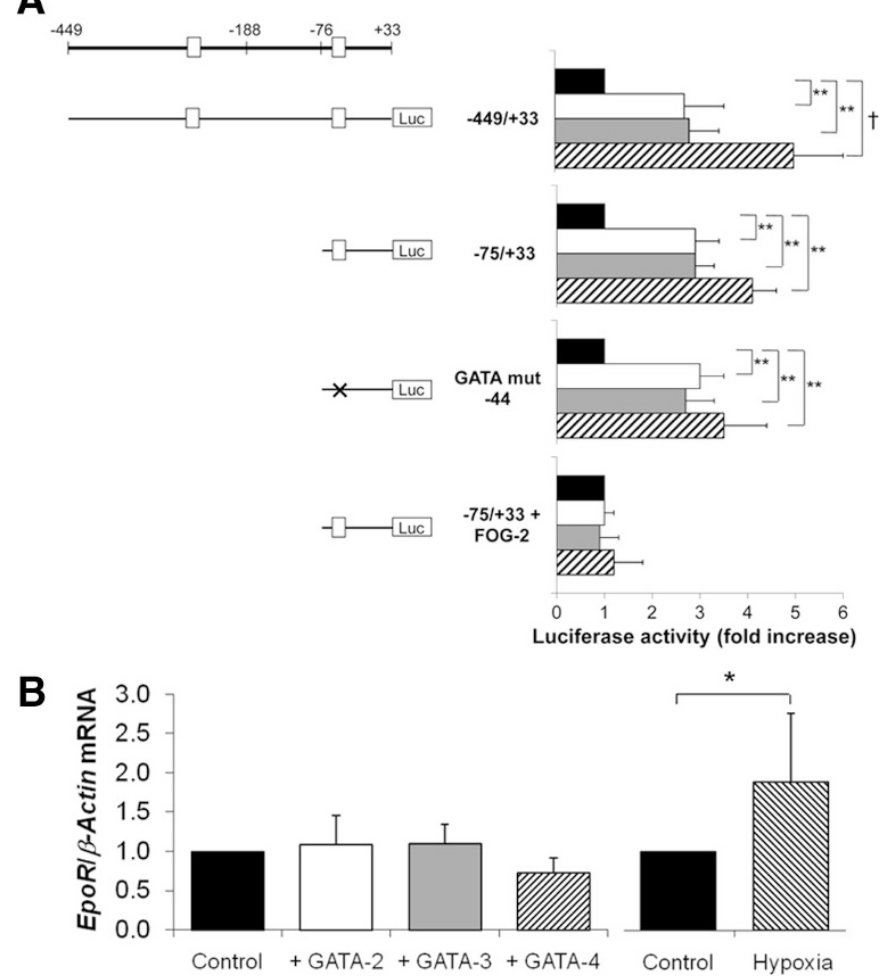

Figure 3. Effect of forced GATA-2, -3 or -4 expression on the EpoR promoter activity and on EpoR expression. (A) SH-SY5Y cells were cotransfected with an EpoR construct and an expression vector (pcDNA3.1/hGATA2/-3 or -4) with or without pcDNA1/hFOG-2 or empty vector. Data present mean $\pm \mathrm{SD}$ from six independent experiments after normalizing the values to the basal level of each reporter construct cotransfected with empty vector (defined as 1). Columns: black, +empty vector; white, +GATA-2; gray, +GATA-3; hatched, +GATA-4. $* * p<0.01 ; \dagger p<0.001$ in student $t$ tests. (B) Real-time PCR analysis of EpoR mRNA expression in cells transfected with pcDNA3.1/hGATA-2/-3 or -4 or cultivated under hypoxia. Data present mean \pm SD from 2 to 3 independent experiments after normalizing the values against $\beta$-actin expression. The expression in control cells was defined as 1 . $* p<0.05$ in student $t$ test. factors, analysis was performed with forced FOG-2 expression. Surprisingly, activation by the GATA factors was inhibited when the cells were additionally transfected with hFOG-2.

Although stable transfections with expression vectors for hGATA-2, -3, and -4 were successful, this did not result in significant changes of endogenous EpoR expression (Fig. 3B). We also checked EpoR expression in SH-SY5Y cells with siRNA-induced downregulation of hGATA-2, -3, or -4. No significant effect on the EpoR expression was found by realtime PCR, although GATA mRNA expression levels were knocked down to about $43-60 \%$ of those in controls (not shown). However, we could achieve a 1.7-1.8 \pm 0.9-fold increase of EpoR transcripts if GATA-2, -3, or -4 were cotransfected with Sp1 (not significant; not shown).

Additionally, we analyzed the effect of hypoxia and rEpo treatment on EpoR mRNA expression. Cultivating SH-SY5Y cells under $2 \% \mathrm{O}_{2}$ for $72 \mathrm{~h}$ resulted in moderate, but significant upregulation of EpoR mRNA (1.9-fold, $p<0.05$ ) (Fig. 3B). Under normoxia, treatment with $5 \mathrm{U} / \mathrm{mL}$ rEpo for $72 \mathrm{~h}$ did not change expression of EpoR or GATA factors (not shown).

Methylation status of the EpoR 5'-flanking region. Because of the discrepancy between activation of EpoR promoter regions by overexpressed GATA transcription factors and their absent effect on endogenous EpoR mRNA expression, we analyzed the sequence with respect to potential methylation sites. There are $20 \mathrm{CpG}$ motifs in the region spanning nt -600 to +149 . The first $\mathrm{CpG}$ is located directly $5^{\prime}$ to the GATA motif in position $-274 /-271$. To test whether methylation plays a role in EpoR expression, we incubated SH-SY5Y cells with different doses of Aza for $72 \mathrm{~h}$, which leads to demethylation of genomic DNA. Indeed, demethylation resulted in a dose dependant increase of EpoR mRNA (Fig. 4).

Next, we analyzed the methylation status of the $20 \mathrm{CpGs}$ in the EpoR 5'-flanking region in SH-SY5Y cells and in four different regions of the human adult CNS (enthorinal, frontal and motor cortex, and cervical spinal cord) (Fig. 5). The analyzed fragment of the EpoR 5'-flanking region showed modest methylation in SH-SY5Y cells, which exhibit the phenotype of fetal neuronal precursors. CpG no. 2, 13, and 14 were methylated in $50 \%$ of clones and two others in $<17 \%$. In the adult brain, methylation slightly increased and showed a different pattern. CpG no. 2 and three others (no. 4, 12, and 20) were methylated in $66-100 \%$ of clones. Several other CpGs were methylated in $<50 \%$ of the clones from enthorinal and frontal cortex specimens.

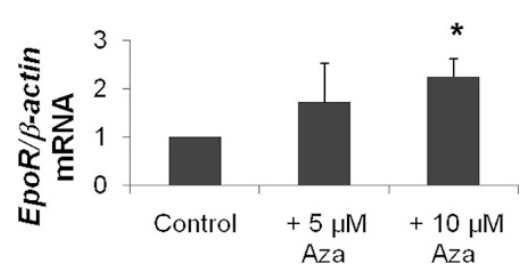

Figure 4. Effect of demethylation of genomic DNA from SH-SY5Y cells with Aza on EpoR mRNA expression measured by real-time PCR (mean \pm $\mathrm{SD}$; three independent experiments after normalizing the values against $\beta$-actin expression). Expression in untreated cells was defined as $1 .{ }^{*} p<0.05$ in ANOVA with post hoc comparison. 
A

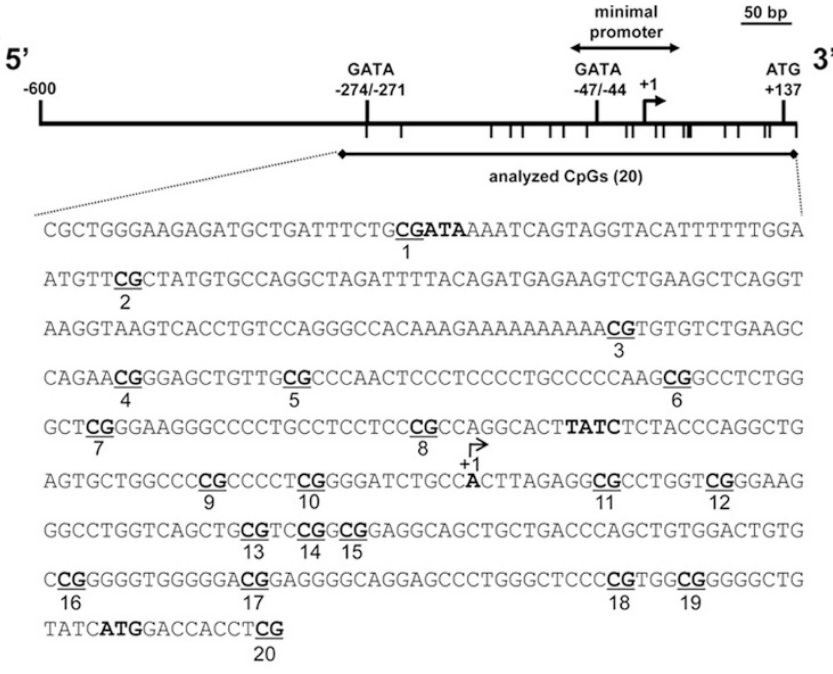

B

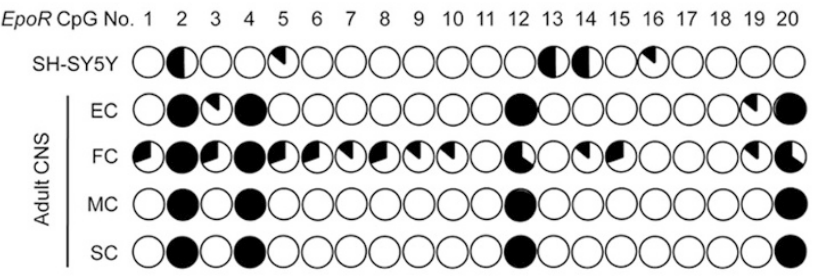

000000

Figure 5. Methylation of human EpoR promoter region. (A) Upper panel shows the structure of EpoR promoter region from nt -600 to +149 . Illustrated are both GATA binding sites, which showed in vitro binding of GATA factors, hematopoietic minimal promoter $(-76 /+33)$, transcription start site $(+1)$ marked with an arrow, and translation start site ATG. Small vertical bars indicate $20 \mathrm{CpG}$ sites. Lower panel shows the analyzed sequence (nt -300 to +149 ). Each CpG site is underlined, in bold, and numbered in order beneath the nucleotide sequence. Both GATA binding sites and translation start site are also in bold. $(B)$ Results of bisulfite sequencing. Circles represent $\mathrm{CpG}$ sites with percentage of methylated clones (number of methylated clones/ 6 analyzed clones $\times 100$ ). $E C$, enthorinal cortex; $F C$, frontal cortex; $M C$, motor cortex; $S C$, spinal cord.

\section{DISCUSSION}

The great potential of rEpo in neuroprotection and/or regeneration may be limited by the developmental downregulation of EpoR expression (6). On the opposite, EpoR expression has been found in different tumors, and concerns in using rEpo to treat anemia during chemotherapy have been raised (1) (http://www.fda.gov/medwatch/safety/2008/epo_DHCP_ 03102008.pdf). This causes more general interest on EpoR regulation in nonhematopoietic cells. Numerous previous studies describing developmental and cellular EpoR expression patterns are inconsistent, because antibodies were used that turned out to be largely unspecific $(19,20)$. However, our analysis confirmed the developmental downregulation of the EpoR in the murine brain (Fig. 1) (4,6). As previously reported for cultured primary neurons, astrocytes, and microglia of various species $(10,21)$, we also detected EpoR mRNA transcripts in these cell types isolated from adult rats (Fig. 1B). Additionally, EpoR was expressed in murine adult NSC (Fig. $1 B$ ), which may be the correlate for Epo-mediated induction of neuronal progenitor differentiation (22).
Among the transcription factors involved in EpoR regulation in hematopoietic cells (23-25), only Sp1 and Wilms tumor suppressor-1 (in its Wt1(-KTS) isoform), but not GATA-1 are expressed in the CNS. Recent data indicate that Wt1(-KTS) does not activate EpoR expression in nonhematopoietic cells (25). The findings that stable transfection of GATA-3 almost doubled the amount of EpoR mRNA and increased EpoR promoter activity in human teratoma-derived neuronal precursor (NT2) cells (3), raised the question whether members of the GATA transcription factor family regulate neuronal EpoR expression.

Our analysis provides novel information on the expression pattern of GATA transcription factors during brain development and in brain-derived primary cell types. Previous analyses of mutant mice with targeted deletions of Gata-2 and Gata-3 indicate that both transcription factors have significant implication in brain development $(26,27)$, whereas disorders in brain development of mutant mice with homozygous Gata-4 deficiency have not been described. However, Gata-4 expression has been reported in migrating gonadotropinreleasing neurons of developing mice (28), and own preliminary data indicate GATA-4 expression in glioblastomaderived cells (Hoene et al., unpublished). In developmental analysis, we show a peak in Gata-2 and Gata-3 mRNA expression at mid gestation, whereas very low Gata-4 transcripts were detected only at E11.5 (Fig. 1A). In addition to previous studies that identified Gata-2 and Gata-3 expression in subtypes of neuronal precursor cells and mature neurons $(26,27,29,30)$, we describe for the first time Gata-2 expression in primary astrocytes (Fig. 1B). In contrast, Gata-3 is expressed both in primary astrocytes and microglia, a novel finding that may have implication in inflammatory processes in the brain. However, lack of Gata-4 transcripts in any primary cell line points to Gata- 4 redundancy in the normal nervous system, even if low transcription was detected in cultured NSC (Fig. 1B).

GATA proteins isolated from SH-SY5Y cells, which express functional EpoR $(17,18)$, bind indeed with a specific pattern to two out of six predicted GATA binding sites (Fig. $2 B$ ). Although all three GATA factors bind to the motif located at nt $-274 /-271$, only GATA-3 and -4 bind to the GATA motif at nt $-47 /-44$, which is located within the minimal hematopoietic EpoR promoter and has been identified as binding motif for GATA-1 $(11,23,31,32)$. In hematopoietic cells a positively regulating domain at nt -76 to +78 , that contains the minimal EpoR promoter, and two negatively regulating domains $(-1050 /-450$ and $+78 /+136)$ were identified (31). Thus GATA factors, expressed in neuronal precursor cells, obviously do not bind to negative regulatory domains within the EpoR 5'-flanking region.

Accordingly, reporter gene assays indicate that GATA-2, -3 , and -4 significantly induce the activity of two EpoR constructs (Fig. 3A). Unexpectedly, stable overexpression had no significant effect on EpoR mRNA expression in SH-SY5Y cells (Fig. 3B), and in an opposite approach, suppression of endogenous GATA expression by siRNAs also failed to change EpoR gene expression. Similar as reported in NT2 cells with stable GATA-3/Sp1 overexpression, we found al- 
most the doubling of EpoR transcripts if SH-SY5Y cells were cotransfected with GATA and Sp1 expressing vectors [(3), C.T. Noguchi, personal communication]. Of note, this effect was not statistically significant. Both NT2 and SH-SY5Y cells responded similarly to hypoxia with a significant 2 -fold induction of EpoR transcription. Interestingly, transfection with FOG-2, an important cofactor for GATA function (33), totally abolished the effect of GATA-2, -3 , and -4 in activating the EpoR reporter gene (Fig. 3A), suggesting a squelching effect. FOG-2 is known to act as suppressor or activator of GATA factors, depending on the regulatory context (33). Thus, our cumulative data do not support a role of GATA factors in directly regulating the EpoR gene expression.

However, demethylation of genomic DNA resulted in a significant upregulation of EpoR mRNA (Fig. 4), leading to the hypothesis that EpoR downregulation during brain development may be due to epigenetic alterations. Within the EpoR $5^{\prime}$-flanking region from $\mathrm{nt}-1779$ to $-606,31 \mathrm{CpGs}$ are located followed by a $330 \mathrm{bp}$ long CpG-free sequence. Another stretch of $19 \mathrm{CpGs}$ is found beginning with one directly at the GATA binding site in position $-274 /-271$ and going up to the translational start site (Fig. 5A). The ATG is also followed by several additional $\mathrm{CpG}$ motifs. Interestingly, the $-300 /+149$ fragment showed a slightly lower methylation rate and a different methylation pattern in SY-SY5Y cells, that exhibit a fetal neuronal phenotype, than in specimens from the human adult brain (Fig. 5B). Within the studied fragment, the methylation may not totally abolish transcriptional activity. For example, $\mathrm{CpG}$ motifs that overlap with $\mathrm{Sp} 1$ binding sites (no. 5-9, 16, and 19) show no or only low methylation, whereas various transcription factors (including members of the Ets family) potentially bind around the $\mathrm{CpG}$ motifs 2, 4, and 12 that are methylated in the adult brain (Fig. 5). Because preliminary data indicate that mutation of the Ets binding motif in position nt $+19 /+24$ induces activity of EpoR reporter constructs (Wallach et al., unpublished), regulatory mechanisms of EpoR expression in the brain may include transcriptional regulation as well as epigenetic silencing.

Acknowledgments. Olaf Hoffmann and Dorette Freyer (Klinik für Neurologie, Charité-Universitätsmedizin Berlin) kindly provided the brain-derived isolated cells. Malte Cremer (Klinik für Neonatologie) performed statistical analysis.

\section{REFERENCES}

1. Arcasoy MO 2008 The non-haematopoietic biological effects of erythropoietin. Br J Haematol 141:14-31

2. Digicaylioglu M, Lipton SA 2001 Erythropoietin-mediated neuroprotection involves cross-talk between Jak2 and NF-kappaB signalling cascades. Nature 412:641-647

3. Yu X, Shacka JJ, Eells JB, Suarez-Quian C, Przygodzki RM, Beleslin-Cokic B, Lin CS, Nikodem VM, Hempstead B, Flanders KC, Costantini F, Noguchi CT 2002 Erythropoietin receptor signalling is required for normal brain development. Development 129:505-516

4. Tsai PT, Ohab JJ, Kertesz N, Groszer M, Matter C, Gao J, Liu X, Wu H, Carmichael ST 2006 A critical role of erythropoietin receptor in neurogenesis and post-stroke recovery. J Neurosci 26:1269-1274

5. Chen ZY, Asavaritikrai P, Prchal JT, Noguchi CT 2007 Endogenous erythropoietin signaling is required for normal neural progenitor cell proliferation. J Biol Chem 282:25875-25883

6. Liu C, Shen K, Liu Z, Noguchi CT 1997 Regulated human erythropoietin receptor expression in mouse brain. J Biol Chem 272:32395-32400
7. Brines M, Grasso G, Fiordaliso F, Sfacteria A, Ghezzi P, Fratelli M, Latini R, Xie QW, Smart J, Su-Rick CJ, Pobre E, Diaz D, Gomez D, Hand C, Coleman T, Cerami A 2004 Erythropoietin mediates tissue protection through an erythropoietin and common beta-subunit heteroreceptor. Proc Natl Acad Sci USA 101:14907-14912

8. Chin K, Yu X, Beleslin-Cokic B, Liu C, Shen K, Mohrenweiser HW, Noguchi CT 2000 Production and processing of erythropoietin receptor transcripts in brain. Brain Res Mol Brain Res 81:29-42

9. Spandou E, Papoutsopoulou S, Soubasi V, Karkavelas G, Simeonidou C, Kremenopoulos G, Guiba-Tziampiri O 2004 Hypoxia-ischemia affects erythropoietin and erythropoietin receptor expression pattern in the neonatal rat brain. Brain Res 1021:167-172

10. Nagai A, Nakagawa E, Choi HB, Hatori K, Kobayashi S, Kim SU 2001 Erythropoietin and erythropoietin receptors in human CNS neurons, astrocytes, microglia, and oligodendrocytes grown in culture. J Neuropathol Exp Neurol 60:386-392

11. Zon LI, Youssoufian H, Mather C, Lodish HF, Orkin SH 1991 Activation of the erythropoietin receptor promoter by transcription factor GATA-1. Proc Natl Acad Sci USA 88:10638-10641

12. Gritti A, Galli R, Vescovi AL 2001 Cultures of stem cells of the central nervous system. In: Fedoroff S, Richardson A (eds) Protocols for Neural Cell Culture. Humana Press, Totowa, New Jersey, pp 173-198

13. Harms C, Lautenschlager M, Bergk A, Freyer D, Weih M, Dirnagl U, Weber JR, Hortnagl H 2000 Melatonin is protective in necrotic but not in caspase-dependent, free radical-independent apoptotic neuronal cell death in primary neuronal cultures. FASEB J 14:1814-1824

14. Ruscher K, Freyer D, Karsch M, Isaev N, Megow D, Sawitzki B, Priller J, Dirnagl U, Meisel A 2002 Erythropoietin is a paracrine mediator of ischemic tolerance in the brain: evidence from an in vitro model. J Neurosci 22:10291-10301

15. Dame C, Sola MC, Lim KC, Leach KM, Fandrey J, Ma Y, Knopfle G, Engel JD, Bungert J 2004 Hepatic erythropoietin gene regulation by GATA-4. J Biol Chem 279:2955-2961

16. Dame C, Kirschner KM, Bartz KV, Wallach T, Hussels CS, Scholz H 2006 Wilms tumor suppressor, Wt1, is a transcriptional activator of the erythropoietin gene. Blood 107:4282-4290

17. Stolze I, Berchner-Pfannschmidt U, Freitag P, Wotzlaw C, Rossler J, Frede S, Acker H, Fandrey J 2002 Hypoxia-inducible erythropoietin gene expression in human neuroblastoma cells. Blood 100:2623-2628

18. Um M, Gross AW, Lodish HF 2007 A "classical" homodimeric erythropoietin receptor is essential for the antiapoptotic effects of erythropoietin on differentiated neuroblastoma SH-SY5Y and pheochromocytoma PC-12 cells. Cell Signal 19:634645

19. Elliott S, Busse L, Bass MB, Lu H, Sarosi I, Sinclair AM, Spahr C, Um M, Van G, Begley CG 2006 Anti-Epo receptor antibodies do not predict Epo receptor expression. Blood 107:1892-1895

20. Kirkeby A, van Beek J, Nielsen J, Leist M, Helboe L 2007 Functional and immunochemical characterisation of different antibodies against the erythropoietin receptor. J Neurosci Methods 164:50-58

21. Morishita E, Masuda S, Nagao M, Yasuda Y, Sasaki R 1997 Erythropoietin receptor is expressed in rat hippocampal and cerebral cortical neurons, and erythropoietin prevents in vitro glutamate-induced neuronal death. Neuroscience 76:105-116

22. Shingo T, Sorokan ST, Shimazaki T, Weiss S 2001 Erythropoietin regulates the in vitro and in vivo production of neuronal progenitors by mammalian forebrain neural stem cells. J Neurosci 21:9733-9743

23. Chin K, Oda N, Shen K, Noguchi CT 1995 Regulation of transcription of the human erythropoietin receptor gene by proteins binding to GATA-1 and Sp1 motifs. Nucleic Acids Res 23:3041-3049

24. Maouche L, Lucien N, Cartron JP, Chretien S 1995 A CCACC motif mediates negative transcriptional regulation of the human erythropoietin receptor. Eur J Biochem 233:793-799

25. Kirschner KM, Hagen P, Hussels CS, Ballmaier M, Scholz H, Dame C 2008 The Wilms' tumor suppressor Wt1 activates transcription of the erythropoietin receptor in hematopoietic progenitor cells. FASEB J 22:2690-2701

26. Zhou Y, Yamamoto M, Engel JD 2000 GATA2 is required for the generation of V2 interneurons. Development 127:3829-3838

27. Lim KC, Lakshmanan G, Crawford SE, Gu Y, Grosveld F, Engel JD 2000 Gata3 loss leads to embryonic lethality due to noradrenaline deficiency of the sympathetic nervous system. Nat Genet 25:209-212

28. Lawson MA, Mellon PL 1998 Expression of GATA-4 in migrating gonadotropinreleasing neurons of the developing mouse. Mol Cell Endocrinol 140:157-161

29. Craven SE, Lim KC, Ye W, Engel JD, de Sauvage F, Rosenthal A 2004 Gata2 specifies serotonergic neurons downstream of sonic hedgehog. Development 131:1165-1173

30. Moriguchi T, Takako N, Hamada M, Maeda A, Fujioka Y, Kuroha T, Huber RE, Hasegawa SL, Rao A, Yamamoto M, Takahashi S, Lim KC, Engel JD 2006 Gata3 participates in a complex transcriptional feedback network to regulate sympathoadrenal differentiation. Development 133:3871-3881

31. Maouche L, Cartron JP, Chretien S 1994 Different domains regulate the human erythropoietin receptor gene transcription. Nucleic Acids Res 22:338-346

32. Chiba T, Ikawa Y, Todokoro K 1991 GATA-1 transactivates erythropoietin receptor gene, and erythropoietin receptor-mediated signals enhance GATA-1 gene expression. Nucleic Acids Res 19:3843-3848

33. Lu JR, McKinsey TA, Xu H, Wang DZ, Richardson JA, Olson EN 1999 FOG-2, a heart- and brain-enriched cofactor for GATA transcription factors. Mol Cell Biol 19:4495-4502 